\title{
Controlled two year follow up of rehabilitation for disorders in the neck and shoulders
}

\author{
Kerstin Ekberg, Bo Björkqvist, Per Malm, Birgitta Bjerre-Kiely, Olav Axelson
}

\begin{abstract}
Objective-To evaluate the effects of an early, active, and multidisciplinary rehabilitation programme for neck and shoulder disorders.
\end{abstract}

Methods-Primary health care and industrial health care of a nonrandomised, controlled, cohort was followed up over two years in a geographically defined area. The cohort consisted of working people who consulted a physician about disorders of the neck or shoulders from 1 August 1988 to 31 October 1989. Criteria for acceptance; not chronic symptoms, patients had sick leave of no more than four weeks. Disorders were not caused by trauma, infections, malignancy, rheumatic diseases, abuse, or pregnancy. 107 people qualified for the study, $87 \%$ were followed up for two years. They were divided into two groups. One group obtained active, multidisciplinary rehabilitation for eight weeks that comprised physical training, information, education, social interaction, and work place visits. Controls were given traditional treatment; physiotherapy, medication, rest, and sick leave. The main outcome measures were: average number of days of sick leave for the two years after rehabilitation, subjective pain on a visual analogue scale, and ratings on seven subscales of the sickness impact profile.

Results-At 12 and 24 months of follow up effects of the active rehabilitation programme did not differ from traditional treatment in any of the outcome measures. New work task $(P<0.05)$ or changed work place $(P<0.001)$ during the follow up period were associated with decreased sick leave, independent of treatment.

Conclusions-Active, multidisciplinary rehabilitation of neck and shoulder disorders was not more effective than traditional treatment. Changed work conditions were associated with decreased sick leave, independent of type of treatment provided.

(Occup Environ Med 1994;51:833-838)

Keywords: neck, rehabilitation, work

Back and neck complaints attract considerable health care resources and reduce work productivity in many countries. Usually the underlying pathology remains unclear and the therapeutic results vary considerably. Controlled studies on efforts of rehabilitation for disorders of the neck and shoulders are rare. A review of rehabilitation studies on patients with back and neck pain showed that out of four studies, the two with the "highest" methodological scores were unsuccessful, and the other two had success in rehabilitation. ${ }^{1}$ In a randomised clinical trial of treatment for neck and back complaints, manual treatment seemed to be slightly better than physiotherapy for the main complaints and physical functioning. ${ }^{2}$ In a cross sectional study Bonsall et al found little effect on sick leave from physiotherapy whereas altered management attitudes to short term sickness absence had a significantly positive effect. ${ }^{3}$ Linton et al compared a group that waited only a short time to see the physician and physiotherapist and a similarly treated group that had to wait longer. The early, active treatment resulted in less sickness absence for "first time" patients only. ${ }^{4}$

Our study focused on the ability to work after early, intense rehabilitation of work related neck and shoulder disorders. Different types of treatment were used in a comprehensive, structured rehabilitation programme, based on the available resources in a well established industrial health care unit. By contrast a comparison group obtained traditional, unstructured treatment of varying intensity. The aim of the study was to determine the magnitude of the supposed health promoting effects of early and multidisciplinary rehabilitation.

\section{Patients and methods}

The study population lived in a semirural community in southern Sweden, where the industrial health care unit and the primary health care unit cover virtually all primary health care needs. The working population belongs to one of the health care units, mainly depending upon geographical location. The industrial health care unit offers health care to those who are employed at companies connected to the health care unit, whereas the primary health care unit is open to anyone in the community. The labour market is characterised by many small manufacturing companies. Piecework is common.

\section{SELECTION OF SUBJECTS}

The subjects were recruited from among the people who consulted a physician for musculoskeletal disorders of the neck, shoulder, 
arm, or upper thorax in either of the health care units from 1 August 1988 to 31 October 1989. To qualify for the study, the person should not have been on continuous sick leave due to the disorder for more than four weeks and should have been in work for at least four of the last six months before seeing the physician. These criteria were set to exclude people who had chronic musculoskeletal problems and subsequently changed lifestyle. Disorders due to or associated with traumatic events or infectious agents, malignancy, rheumatic diseases (affecting the joints, such as arthritis), abuse, and pregnancy were excluded, whereas soft tissue rheumatism was accepted. Cervical spondylosis, as verified by $x$ ray film, was accepted only as a supplementary diagnosis to the main diagnoses of cervical syndrome and tension neck syndrome. All subjects were in work and aged between 18 and 59. They had been employed in their present occupation for at least two months, and they had worked $100 \%$ of their employment time for at least four of the last six months. The subjects were clinically examined by the same physician and physiotherapist before treatment and after 12 months. Diagnoses were set according to criteria described by Waris et al. ${ }^{5}$

\section{ASSIGNMENT OF TREATMENT}

Subjects who consulted the physician at the industrial health care unit were assigned to an active rehabilitation programme, whereas subjects at the primary health care unit obtained traditional treatment.

\section{Active rehabilitation programme}

All people who consulted the physician at the industrial health care unit participated in the active rehabilitation programme for eight weeks to improve strength, mobility, and local endurance in the neck, shoulders, and upper extremities. More general training was also included to improve general health and to make the training more attractive and varied. The patients trained for two hours a day, four days a week for eight weeks. Usually small groups of five to eight patients trained with one or two physiotherapists. When necessary, training was adjusted to the capacity of the individual. As well as the physical training programme the patients were given education in how to promote health, and they participated in neck and back schools. They were also given information on ergonomics, physical training in general, and the health effects of smoking and nutrition. Insurance matters and vocational training were also discussed. Some social interaction in the groups took place. The industrial health care unit visited all the workplaces and attempts were made to make improvements. All patients were at least on $50 \%$ sick leave-that is, they worked for half time at the most, independent of the degree of severity of the disorder, to enable them to follow the active rehabilitation programme.

Traditional treatment

All patients who belonged to the primary health care unit were treated in a traditional way. The treatment of these patients was more passive and did not comprise group activities, education, or efforts to improve the work conditions, but they were offered physiotherapy, medication, or other types of treatments along with rest or sick leave as considered necessary by the physicians. Twelve of the patients in this group were given non-steroidal anti-inflammatory drugs (NSAID) during the first year; 17 patients had physiotherapy, with the number of treatment sessions ranging from four to 25. The type of physiotherapy varied, a few patients had manual treatment, nine had physiotherapy with ultrasound treatment or only ultrasound, eight had massage, two had transcutaneous nerve stimulation (TENS), and three had acupuncture. Several patients went through more than one type of treatment.

\section{OUTCOME MEASURES}

\section{Sick leave}

Information on sick leave was obtained from the Swedish social insurance office. The number of days of sick leave due to musculoskeletal disorders was noted for each patient for the entire year before the treatment, and for each quarter of the year during the follow up period.

\section{Symptoms and pain}

Before the treatment all patients answered a modified version of the Nordic questionnaire on musculoskeletal symptoms, stating whether they had had symptoms during the past six months. ${ }^{6}$ The subjects also rated their present subjective pain on a visual analogue scale that ranged between 1 (no pain at all) and 10 (unbearable pain). Background factors-that is, age, sex, ethnic background, family situation, smoking habits, and exercise habits-were included. The same questionnaire was answered after 12 months at a clinical check up, and a shortened version was posted to the patients after 24 months.

\section{Health related behaviour}

Before treatment and after 12 months, all patients answered a shortened version of the sickness impact profile, which is a measure of health related behaviour. ${ }^{7}$ The original version comprised 12 scales, but only seven scales (mobility, alertness behaviour, emotional behaviour, social interaction, work, home management, and recreational pastimes) were used in our study.

\section{Working conditions}

Each patient was interviewed about changes in work task and work place at the clinical check up after 12 months.

\section{SUBJECTS}

\section{Active rehabilitation group}

A total of 61 patients qualified for the study at the industrial health care unit and were assigned to the active rehabilitation programme. Out of these $47 \%$ had tension neck 
syndrome, $26 \%$ had humeral tendinitis, and $17 \%$ had cervical syndrome or rhizopathy. The remaining $10 \%$ were assigned to three other diagnoses within the neck and shoulders. During the follow up period five patients became pregnant, one moved and two did not fulfil the training programme. Hence, 53 patients $(87 \%)$ participated for the 24 months of the programme.

\section{Traditional rehabilitation group}

A total of 46 patients qualified for the study at the primary health care unit and were assigned to traditional treatment. Out of these, $40 \%$ had tension neck syndrome, $28 \%$ had humeral tendinitis, $20 \%$ had cervical syndrome or rhizopathy, and $12 \%$ were assigned to three other diagnoses. During the follow up period four patients became pregnant, one moved, and one did not answer the questionnaires. Hence, after 24 months 40 patients $(87 \%)$ remained as controls.

\section{STATISTICAL ANALYSIS}

As the active rehabilitation programme comprised at least $50 \%$ sick leave for eight weeks the first three months were left out in the comparisons between the groups. Sick leave data and the sickness impact profile were compared with the Wilcoxon rank sums test. The log rank test and the Wilcoxon test in the life test procedure of the SAS statistical package were used to compare the rate of return to work during the follow up periodthat is, an evaluation corresponding to survival analysis was performed where days of sick leave until return to work were considered. Pain was compared with the Student's $t$ test. Two sided tests were used in all comparisons. Calculations of Mantel-Haenszel adjusted relative risks for cohort studies (RR) and confidence intervals (CIs) were used. ${ }^{8}$

\section{Results}

The age distribution was similar in the two groups. The proportion of parents who had small children, immigrants, and blue collar workers was higher in the active rehabilitation group than in the control group. Frequent exercise ( $>5 \mathrm{~h} /$ week) was more common in the active rehabilitation group (table 1 ).

In spite of the difference between the

Table 1 Demographic data and lifestyle indicators of the group in active rehabilitation and of the control group

\begin{tabular}{lll}
\hline & $\begin{array}{l}\text { Active } \\
\text { rehabilitation } \\
(n=53)\end{array}$ & $\begin{array}{l}\text { Traditional } \\
\text { rehabilitation } \\
(n=40)\end{array}$ \\
\hline Age: & & \\
$\quad$ Mean (SD) & $38(10)$ & $40(12)$ \\
Median (range) & $39(20-54)$ & $41(18-57)$ \\
Sex, F (\%) & 87 & 70 \\
Have children & & \\
<12 y (\%) & 42 & 35 \\
Immigrant (\%) & 34 & 23 \\
Smokers (\%) & 55 & 68 \\
Exercise >5 h/week (\%) & 16 & 3 \\
Exercise rarely or never (\%) & 54 & 46 \\
Type of employment (\%): & 91 & 55 \\
$\quad$ Blue collar & 9 & 43 \\
Service, health care & 9 & 2 \\
Others & 0 & \\
\hline
\end{tabular}

groups in the proportion of blue collar workers, data from the questionnaire on ergonomic and organisational work conditions showed only minor differences between the groups. The groups were compared for seven factors that described ergonomic work conditions (uncomfortable sitting position, uncomfortable standing position, work with lifted arms, repetitive movements demanding precision, monotonous work position, frequent light lifting, physically demanding work) and eight factors that described organisational and psychosocial work conditions (time pressure, demands on attention, work planning, job security, job constraints, work role ambiguity, quality of work content, and social climate). The questionnaire was fully described by Ekberg et al. ${ }^{9}$ The active rehabilitation group rated themselves as having worse work conditions with regard to monotonous work position $(P<0.001)$ and quality of work content $(P<0.01)$, whereas the traditional rehabilitation group indicated worse work planning $(P<0.01)$. There were no significant differences between the groups in the remaining 12 factors that described work conditions.

\section{SICK LEAVE}

There were no significant differences in sick leave between the groups during the year preceding treatment. The active rehabilitation group had a mean (SD) of 28 (34) days of sick leave, and the traditional rehabilitation group had a mean (SD) of 25 (34) days of sick leave. Three patients in each group had no days of sick leave at all due to musculoskeletal disorders in the year before the study.

The figure shows the mean (95\% CIs) number of days of sick leave from musculoskeletal disorders for each quarter of the year during the follow up period. As mentioned, the number of days of sick leave in the active rehabilitation group was high during the rehabilitation period ( $0-3$ months) to permit participation in the programme. After the rehabilitation period the sick leave due to

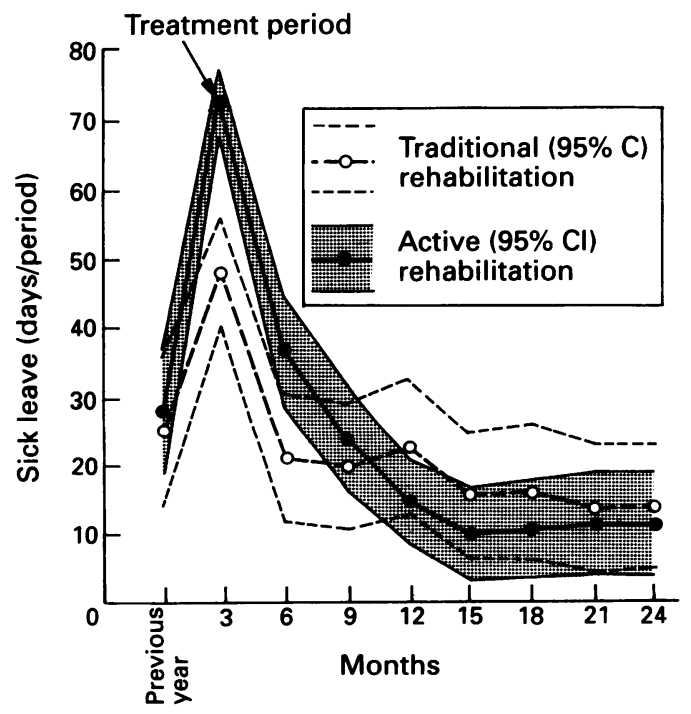

Mean (95\% CI) number of days of sick leave with musculoskeletal disorders during the year preceding treatment and during the follow up period. 
musculoskeletal disorders decreased. The group that had traditional treatment had a faster return to work at the beginning of the follow up period. After 90 days, $21 \%$ in the active and $63 \%$ in the traditional rehabilitation group were back to work $(P=0.04)$. After 210 days slightly more than $80 \%$ in both groups were working. In total, however, the active rehabilitation group had significantly more days of sick leave for the first year than the control group even when the first quarter of the year was excluded $(P=0.05)$. After 24 months the difference between the groups had disappeared $(P=0 \cdot 70)$.

Possible differences in responsiveness to treatment due to age $(<40 v \geqslant 40)$, sex, ethnic background, and the three work environment factors that differed between the groups (monotonous work positions, quality of work content, and work planning) were considered by multiple linear regression with the $\log$ of sick leave as the dependent variable. At the 12 month follow up, the significant predictors of increased sick leave were quality of work content $(P=0.01)$ and being an immigrant $(P=0.003)$. At the 24 month follow up two significant predictors remained, namely being an immigrant $(P=0.01)$ and older age $(P=0.005)$.

\section{SYMPTOMS AND PAIN}

The prevalence of subjective symptoms in the neck and shoulders, as rated in the questionnaire, was unaffected by type of treatment (table 2). Similarly, the groups did not differ in average pain ratings on a visual analogue scale either before treatment, or at any of the follow up measurements (table 2). Both groups improved between the measurement before treatment and that at 12 months

Table 2 Average pain ratings on a visual analogue scale and prevalence of symptoms in the neck and shoulders with time

\begin{tabular}{cll}
\hline & $\begin{array}{l}\text { Active } \\
\text { rehabilitation } \\
(n=53) \text { mean }(S D)\end{array}$ & $\begin{array}{l}\text { Traditional } \\
\text { rehabilitation } \\
(n=40) \text { mean }(S D)\end{array}$ \\
\hline Pain: & & \\
$(0)$ & $6 \cdot 1(1 \cdot 6)$ & $6 \cdot 0(1 \cdot 3)$ \\
$(12)$ & $5 \cdot 3(2 \cdot 0)$ & $5 \cdot 4(1 \cdot 1)$ \\
$(24)$ & $5 \cdot 0(2 \cdot 2)$ & $4 \cdot 8(2 \cdot 1)$ \\
Symptoms (\%): & 94 \\
$(0)$ & 94 & 95 \\
$(12)$ & 92 & 84 \\
$(24)$ & 89 & 94 \\
\hline
\end{tabular}

$(0)=$ before treatment; $(12)=$ after 12 months; $(24)=$ after 24 months.

Table 3 Distribution of patients with increased and decreased sick leave during the second year of follow up into exposure categories

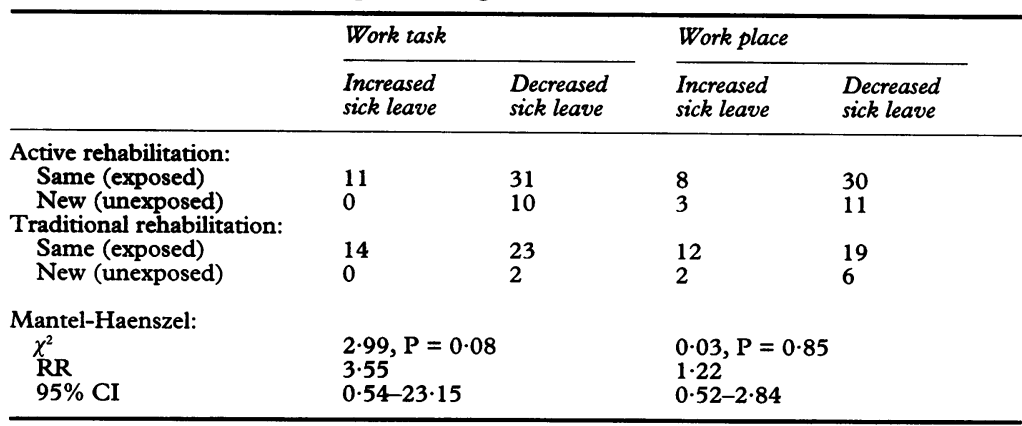

(active rehabilitation group: difference 0-12: $P=0 \cdot 04$, traditional rehabilitation group: difference $0-12: P=0.001)$. There were no within group improvements between the 12 month and the 24 month follow ups. Multiple regression analysis showed that with background variables and the three work environment factors as predictors and pain as a dependent variable being an immigrant was a significant predictor of pain at both the 12 month follow up $(P=0.04)$ and at the 24 month follow up $(P=0.02)$.

\section{HEALTH RELATED BEHAVIOUR}

Mean ratings on the sickness impact profile scales did not differ between the groups before treatment or at the 12 month follow up. Both groups improved between the measurement before treatment and that at 12 months in the scales that referred to work ( $P=0.001$ for both groups), and home management ( $P=0.05$ for both groups). The active rehabilitation group also improved in mobility during the follow up period $(P=0.05)$.

\section{CHANGED WORK CONDITIONS}

Patients who changed work task or place during the first year (non-exposed) were compared for sick leave during the second year with those who had unchanged work conditions (exposed). Patients with increased sick leave were compared with those with unchanged or decreased sick leave during the second year, and were stratified by type of rehabilitation obtained. Unchanged work task (table 3 ) resulted in a relative risk of $3.6(95 \%$ CI 0.5-23.2). The relative risk from unchanged work place was $1.2(95 \% \mathrm{CI}$ $0 \cdot 5-2 \cdot 8)$. As the outcome measure "change in sick leave" was dichotomised in the MantelHaenszel analysis, some sensitivity in the analysis may have been lost, so an analysis of variance that fully used the variation in sick leave data, gave significant effects of changed work task $(f=4.5, P=0.04)$ and of changed work place $(f=13.8, P<0.001$, table 4$)$. Subjects with and without new work conditions did not differ in sick leave before treatment.

In the active rehabilitation group the mean (SD) number of days of sick leave for those with unchanged work task was 98 (90, maximum 323) and for those with changed work task 121 (96, maximum 319). In the traditional rehabilitation group, which only had two patients with changed work task, those patients with unchanged work conditions had 78 (112, maximum 365) days of sick leave,

Table 4 Results of analysis of variance on effects of new work tasks and new work place on changes in sick leave during the second year

\begin{tabular}{llrl}
\hline Source & $D F$ & \multicolumn{1}{l}{$f$} & P valve \\
\hline Model & 5,83 & $4 \cdot 13$ & 0.002 \\
Treatment group & 1 & $0 \cdot 12$ & $0 \cdot 74$ \\
New work task & 1 & $4 \cdot 45$ & 0.04 \\
New work place & 1 & $13 \cdot 78$ & 0.0004 \\
Treatment group $\times$ work task & 1 & 0.73 & 0.39 \\
Treatment group $\times$ work place & 1 & 1.01 & 0.32 \\
\hline
\end{tabular}


and those with changed work task had 107 (47, maximum 140 ).

The mean (SD) number of days of sick leave in the active rehabilitation group for those with unchanged work place during the first year of follow up was 94 (90, maximum 323) and for those who changed their work place 126 (91, maximum 323). In the traditional rehabilitation group the corresponding numbers were 60 (88, maximum 365) for unchanged work places and 154 (156, maximum 365) for changed work places.

Hence, those patients who changed their work conditions on average had more days of sick leave in the first year. The range of days of sick leave was large, independent of changed or unchanged work conditions, and data do not provide unequivocal support for the view that those patients with higher sickness absence changed their work conditions.

\section{Discussion}

The rehabilitation programme applied in this study did not decrease the rate of sick leave but rather delayed return to work. The relatively broad CIs around the estimates of days of sick leave (figure) indicate that larger study groups would have improved the informative value. ${ }^{10}$ Economic constraints hindered a continuation of the study, and power calculations were performed to determine the risk of $\beta$ error in the study. ${ }^{11}$ About 100 patients, as in this study, seem to suffice for a power of $0.75-0.80$ given an $a$ of $0.90-0.95$, an expected prevalence of $60 \%$ in the control group, and $30-40 \%$ in the treated group. Hence, the study may be considered reasonably large to properly elucidate the efficacy of this type of rehabilitation.

One possible explanation for the findings that the more comprehensive active rehabilitation programme had no effect is that the active rehabilitation programme required at least $50 \%$ sick leave so that the patients may have acquired a "sick role" during the treatment period. Similar findings were made by Deyo et al in a study on low back pain. ${ }^{12}$ In their study, return to work was significantly delayed by more days of bed rest.

Ben-Sira in a discussion of the difficulties in readjusting disabled people, suggests that assistance by rehabilitation agencies is more likely to result in dependence than in readjustment. ${ }^{13}$ By analogy with his reasoning, the patients in our traditional treatment group, who were left to depend on their own decisions and initiatives, may thereby have been able to cope better.

Kristensen suggests that sickness absence, a complex outcome measure, should be regarded as a coping behaviour that reflects the perception of health in relation to demands and strain of the job. ${ }^{14}$ Melamed et al found sickness absence to be related to subjective stress in the work place. ${ }^{15}$ Subjects who changed work task during the follow up period had a significantly more positive development with regard to sick leave. As more subjects in the active rehabilitation pro- gramme changed work task or work place, the active rehabilitation efforts may be considered partly successful in this respect.

The sickness impact profile was chosen as an instrument for measuring functional health state. ${ }^{7}$ This questionnaire has previously been used in back pain trials. ${ }^{12}{ }^{16-18}$ In our study the scores before treatment did not differ much from the scores of the general population in five scales. ${ }^{19}$ Scores from scales that measured home management and work indicated worse health behaviour for the patients in our study than in the general population. As both treatment groups improved in these scales during the follow up period the two types of rehabilitation did not differ in effect. Possibly time, as well as rehabilitation efforts, may have been influential on a positive outcome. Results for the pain ratings were similar; both groups improved during the first year.

As women and immigrants have more musculoskeletal disorders in the neck and shoulders, ${ }^{920}$ one presumption was that these groups would gain more from the multifactorial treatment programme. The multiple regression analyses on outcome measures did not support this assumption. Rather, as women and immigrants to a large extent keep those jobs that are characterised by a low quality of work content, ${ }^{20}$ it is likely that the prognosis for a successful rehabilitation of these groups to a large extent depends on improved work conditions.

The methodological problems involved in long term assessment studies are considerable. A crucial point is whether the treated group and the controls are comparable in essential respects. Measurement of exposure in epidemiological studies of musculoskeletal disorders is always a problem. In general it is agreed that the occupational title is a very rough measure, as each occupational title comprises many different work tasks. The two groups in this study differed in type of occupation, as there was a higher proportion of blue collar workers in the actively rehabilitated group. Both groups had answered an extensive questionnaire on ergonomic and organisational work conditions before treatment. The questionnaire data were considered to provide more sensitive information on exposure conditions than did the occupational title. As the two groups differed in only three out of 15 exposure factors, the risk of major differences in work conditions was judged to be small. Possible confounding effects of work conditions and background characteristics on the outcome were analysed in multiple regression analysis. Sick leave during the first 12 months was affected by quality of work content. This supports the hypothesis that improved work conditions are crucial for successful rehabilitation of work related musculoskeletal disorders.

No differences before treatment were found between the two groups in any of the outcome measures. Therefore, the risk of selection bias for severity of disease was considered to be small. Control groups with placebo or passive treatment tend to involve themselves in other 
treatments beyond the control of the researchers, ${ }^{221}$ which may affect the outcome. In our study several patients in the traditional rehabilitation group obtained different types of treatment during the follow up period. The controls were therefore not untreated, but were treated differently from the study patients. The effect of a fairly costly and intensive intervention programme was clearly no better than "normal" treatment.

To summarise, the study describes a comparison of two rehabilitation approaches to neck and shoulder disorders. Efforts were made to control for selection bias between the two rehabilitation groups for both exposure and severity of disease. The groups differed in occupational titles, although their ratings in a questionnaire on ergonomic and organisational work conditions were similar in most respects. The groups did not differ before treatment in the measures of outcome. This indicates that severity of disease was similar. The active, multifactorial rehabilitation programme of neck and shoulder disorders did not decrease sick leave compared with more passive, traditional rehabilitation, but changed work conditions were associated with decreased sick leave, independent of type of treatment given.

This study was supported by the Swedish Work Environment Fund.

1 Koes BW, Assendelft WJJ, van der Heijden GJMG, Bouter LM, Knipschild PG. Spinal manipulation and mobilisation for back and neck complaints: a blinded review. BMF 1991;303:1298-303.

2 Koes BW, Bouter LM, van Mameren H, Essers AHM, Verstegen GMJR, Hofhuizen DM, et al. Randomised clinical trial of manipulative therapy and physiotherapy for persistent back and neck complaints: results of one year follow up. $B M \mathcal{F} 1992 ; 304: 601-5$.

3 Bonsall JL, Squier JEO, Baron CA, Parker G. Effect of physiotherapy on sickness absence in industry: a comparative study. $\mathcal{F}$ Soc Occup Med 1991;41:176-80.

4 Linton SJ, Hellsing A-L, Andersson D. A controlled study of the effects of an early intervention on acute musculoskeletal pain problems. Pain 1993;54:353-9.
5 Waris P, Kuorinka I, Kurppa K, Luopajärvi T, Virolainen $\mathrm{M}$, Pesonen $\mathrm{K}$, et al. Epidemiologic screening of occupational neck and upper limb disorders: methods and criteria. Scand $f$ Work Environ Health 1979;5:25-38.

6 Kourinka I, Jonsson B, Kilbom $\AA$, Vinterberg H Biering-Sorensen F, Andersson G, Jorgensen $\mathbf{K}$ Standardised Nordic questionnaires for the analysis of musculoskeletal symptoms. Applied Ergonomics 1987;3 233-7.

7 Bergner M, Bobbitt RA, Carter WB, Gilson BS. The sickness impact profile: development and final revision of a health status measure. Med Care 1981;19:787-805.

8 Kleinbaum DG, Kupper LL, Morgenstern H. Epidemiologic research: principles and quantitative methods. Belmont, CA: Wadsworth, 1982.

9 Ekberg K, Björkqvist B, Malm P, Bjerre-Kiely B, Karlsson $M$, Axelson O. Cross-sectional study of risk factors for symptoms in the neck and shoulder area. Ergonomics 1994 (in press).

10 Ahlbom A, Axelson A, Stottrup Hansen E, Hogstedt C, Jensen UJ, Olsen J. Interpretation of "negative" studies in occupational epidemiology. Scand $f$ Work Environ Health 1990;16:153-7.

11 Fleiss JL. Statistical methods for rates and proportions. 2nd ed. New York: John Wiley, 1981.

12 Deyo RA, Diehl AK, Rosenthal M. How many days of bed rest for acute low back pain? $N$ Engl F Med 1986;315: 1064-70.

13 Ben-Sira $Z$. Disability, stress and readjustment: the function of the professional's latent goals and affective behavior in rehabilitation. Soc Sci Med 1986;23:43-55.

14 Kristensen TS. Sickness absence and work strain among Danish slaughterhouse workers: an analysis of absence from work regarded as coping behaviour. Soc Sci Med from work rega

15 Melamed S, Luz J, Najenson T, Jucha E, Green M Ergonomic stress levels, personal characteristics, accident occurrence and sickness absence among factory workers. Ergonomics 1989;32:1101-10.

16 Deyo RA. A controlled trial of transcutaneous nerve stimulation (TENS) and exercise for chronic low back pain. N Engl f Med 1990;322:1627-34.

17 Follick MJ, Smith TW, Ahern DK. The sickness impact profile: a global measure of disability in chronic low back pain. Pain 1985;21:67-76.

18 Koes BW, Bouter LM, van Mameren $H$, Essers AHM, Verstegen GMJR, Hofhuizen DM, et al. The effectiveness of manual therapy, physiotherapy, and treatment by ness of manual therapy, physiotherapy, and treatment by the general practitioner for nonspecific back and neck 28-35.

19 Sullivan M, Ahlmén M, Bjelle A. Health status assessment in rheumatoid arthritis. I. Further work on the validity of the sickness impact profile. If Rheumatol 1990;17: 439-47.

20 Ekberg K, Björkqvist B, Malm P, Bjerre-Kiely B, Karlsson $M$, Axelson $\mathrm{O}$. Case-control study of risk factors for disease in the neck and shoulder area. Occup Environ Med 1994;51:262-6.

21 Linton SJ, Bradley LA. An 18-month follow-up of a secondary prevention programme for back pain. Help and hindrance factors related to outcome maintenance. Clin $\mathcal{f}$ Pain 1992;8:227-36. 